\title{
Robust adaptive simultaneous state and fault estimation for nonlinear systems: Application to an aerodynamical system
}

\author{
Mariusz Buciakowski ${ }^{1}$, Marcin Witczak ${ }^{1}$, Vicenç Puig ${ }^{2}$, Damiano Rotondo ${ }^{2}$, Fatiha Nejjari ${ }^{2}$
}

\begin{abstract}
The paper is concerned with the task of robust adaptive fault estimation and an unknown input decoupling for nonlinear systems using a quadratic boundedness approach. In particular, the fault estimation strategy and decoupling of the unknown input is based on an unknown input observer. The above methods are used to describe a robust fault and state observer problem by a set of linear matrix inequalities, which are efficiently handled by freely available solvers. The proposed approach allows obtaining a feasible set of joint state and fault estimation errors. Based on this knowledge, the confidence intervals of the system state and actuator fault, which supports diagnostic decisions, are proposed. The final part of the paper presents an illustrative example concerning an aerodynamical twin-rotor system, which exhibits the performance of the proposed approach.
\end{abstract}

\section{INTRODUCTION}

The problem of fault estimation, which can also be perceived as the estimation of an unknown input has received considerable attention during the last three decades [1], [2]. Many works have presented different strategies for fault estimation, in particular: two-stage Kalman filter [3], minimum variance input and state estimator [4], adaptive estimation [5], sliding mode high-gain observers [6], and $\mathcal{H}_{\infty}$ approach [7], max-plus algebra [8]. Although some approaches for nonlinear systems have been proposed as high gain observer for Lipschitz systems [9], geometric approach [10] however, the fault estimation for problem nonlinear system is still an open issue of paramount importance.

This paper extends the approach developed by the authors in [11] where the objective was to determine an optimal state and fault estimation in the sense of $\mathcal{H}_{\infty}$ norm. In this work, a design strategy that additionally provides confidence intervals overbounding the estimates are consistent with the disturbances and measurement noises. The observer design procedure is based on the quadratic boundedness approach [12], which allows using powerful tools in the form of linear matrix inequalities (LMIs) whose solution is easily generated using available solvers.

The paper is organised as follows. Section II presents preliminaries regarding the problem being considered. In

\footnotetext{
${ }^{1}$ Institute of Control and Computation Engineering, University of Zielona Góra, ul. Podgórna 50, 65-246 Zielona Góra, Poland. M.Witczak, M.Buciakowski@issi.uz.zgora.pl

${ }^{2}$ Automatic Control Department, Universidad Politécnica de Cataluña, Rambla Sant Nebridi, 10, 08222 Terrassa, Spain. vicenc.puig, damiano.rotondo@upc.edu

fatiha.nejjari@upc.edu
}

Section III, the robust fault estimation approach is proposed while in Section IV, the unknown input observer design procedure is described. Section V presents the strategy for calculating confidence intervals overbounding the unknown state and fault. Section VI presents the application of the proposed approach to a Twin Rotor MIMO System (TRMS), showing its effectiveness and performance. Finally, the last section concludes the paper.

\section{PREliminaries}

The system under consideration is given by a nonlinear discrete-time state-space form

$$
\begin{aligned}
\boldsymbol{x}_{k+1} & =\boldsymbol{A} \boldsymbol{x}_{k}+\boldsymbol{B} \boldsymbol{u}_{k}+\boldsymbol{D} \boldsymbol{d}_{k}+\boldsymbol{g}\left(\boldsymbol{x}_{k}, \boldsymbol{u}_{k}\right) \\
& +\boldsymbol{B} \boldsymbol{f}_{k}+\boldsymbol{W}_{1} \boldsymbol{w}_{k}, \\
\boldsymbol{y}_{k} & =\boldsymbol{C} \boldsymbol{x}_{k}+\boldsymbol{W}_{2} \boldsymbol{w}_{k},
\end{aligned}
$$

where $\boldsymbol{x}_{k} \in \mathbb{R}^{n}$ denotes the state, $\boldsymbol{u}_{k} \in \mathbb{R}^{r}$ is the control input, $\boldsymbol{y}_{k} \in \mathbb{R}^{m}$ denotes the output, $\boldsymbol{f}_{k} \in \mathbb{R}^{s}$ expresses the fault, $\boldsymbol{d}_{k} \in \mathbb{R}^{q}$ stands for unknown input disturbance vector, $\boldsymbol{w}_{k}$, stands for system disturbance, measurements noises, etc, $\boldsymbol{g}\left(\boldsymbol{x}_{k}, \boldsymbol{u}_{k}\right)$ is non-linear function and is assumed to be differentiable with respect to $\boldsymbol{x}_{k}$ and $\boldsymbol{u}_{k}, \boldsymbol{W}_{1} \in \mathbb{R}^{n \times n}$, $\boldsymbol{W}_{2} \in \mathbb{R}^{m \times n}$ stand for distribution matrices, $\boldsymbol{w}_{k} \in \mathbb{R}^{n}$ is a an exogenous disturbance vector. For the purpose of this work, the following assumptions (AS) are considered:

AS 1: There exist a matrix $M \in \mathbb{M}$ such that

$$
\begin{aligned}
& (\boldsymbol{g}(\boldsymbol{a}, \boldsymbol{u})-\boldsymbol{g}(\boldsymbol{b}, \boldsymbol{u}))^{T}(\boldsymbol{a}-\boldsymbol{b}) \leq \\
& (\boldsymbol{a}-\boldsymbol{b})^{T} \boldsymbol{M}^{T}(\boldsymbol{a}-\boldsymbol{b}) .
\end{aligned}
$$

AS 2: There exist a matrix $M \in \mathbb{M}$ such that

$$
\begin{aligned}
& (\boldsymbol{g}(\boldsymbol{a}, \boldsymbol{u})-\boldsymbol{g}(\boldsymbol{b}, \boldsymbol{u}))^{T}(\boldsymbol{g}(\boldsymbol{a}, \boldsymbol{u})-\boldsymbol{g}(\boldsymbol{b}, \boldsymbol{u})) \\
& \leq(\boldsymbol{a}-\boldsymbol{b})^{T} \boldsymbol{M}^{T} \boldsymbol{M}(\boldsymbol{a}-\boldsymbol{b}) .
\end{aligned}
$$

AS 3: The ellipsoidal set is as follow

$$
\begin{aligned}
\boldsymbol{\varepsilon}_{k} & =\boldsymbol{f}_{k+1}-\boldsymbol{f}_{k}, \\
\varepsilon_{k} \in \mathcal{E}_{\varepsilon}, \mathcal{E}_{\boldsymbol{\varepsilon}} & =\left\{\boldsymbol{\varepsilon}: \boldsymbol{\varepsilon}^{T} \boldsymbol{Q}_{\boldsymbol{\varepsilon}} \varepsilon \leq 1\right\}, \boldsymbol{Q}_{\boldsymbol{\varepsilon}} \succ 0 .
\end{aligned}
$$

AS 4: Rank condition is satisfied

$$
\operatorname{rank}(\boldsymbol{D})=\operatorname{rank}(\boldsymbol{C D})=q, \quad q \leq m .
$$

Assumption 1 extends the idea of one-sided Lipschitz condition [13]. Assumption 2 extends the usual Lipschitz condition, while Assumption 3 is required to attain a suitable fault 
estimation quality. Finally, it is assumed that system (1)-(2) is observable [4], [2] and the condition from Assumption 4 is satisfied. Let the function $\boldsymbol{g}\left(\boldsymbol{x}_{k}, \boldsymbol{u}_{k}\right)$ be continuous and differentiable. Using the Differential Mean Value Theorem (DMVT) [14] it is possible to show that:

$$
\boldsymbol{g}(\boldsymbol{a}, \boldsymbol{u})-\boldsymbol{g}(\boldsymbol{b}, \boldsymbol{u})=\boldsymbol{M}_{x, u}(\boldsymbol{a}-\boldsymbol{b}),
$$

with

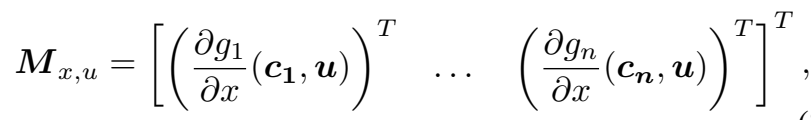

where $\boldsymbol{c}_{1}, \ldots, \boldsymbol{c}_{n} \in \operatorname{Co}(\boldsymbol{a}, \boldsymbol{b}), \boldsymbol{c}_{i} \neq \boldsymbol{a}, \boldsymbol{c}_{i} \neq \boldsymbol{b}, i=1, \ldots, n$. Based on the fact that $\boldsymbol{x}_{k}$ is bounded, i.e., $\boldsymbol{x}_{k} \in \mathbb{X}$, it is possible to show

$$
\bar{a}_{i, j} \geq \frac{\partial g_{i}(\boldsymbol{x}, \boldsymbol{u})}{\partial x_{j}} \geq \underline{a}_{i, j}, \quad i=1, \ldots, n, \quad j=1, \ldots, n,
$$

where $\bar{a}_{i, j} \geq \underline{a}_{i, j}$ are known bounds. Thus, it is clear that there exists a matrix $M \in \mathbb{M}$ such that

$$
\mathbb{M}=\left\{M \in \mathbb{R}^{n \times n} \mid \bar{a}_{i, j} \geq m_{i, j} \geq \underline{a}_{i, j}, i, j=1, \ldots, n,\right\} .
$$

It is worth mentioning that, if $\boldsymbol{M}^{T} \boldsymbol{M}=\gamma^{2} \boldsymbol{I}$, then $A s$ sumption 2 becomes a usual Lipschitz condition [15], [16], with $\gamma$ being a Lipschitz constant. This property makes the proposed strategy more general than those presented in the literature [15]. For the future analysis, let us remind the following lemma [17]:

Lemma 1: The following statements are equivalent:

1) There exist $\boldsymbol{X} \succ 0$ and $\boldsymbol{W} \succ 0$ such that

$$
\boldsymbol{V}^{T} \boldsymbol{X} \boldsymbol{V}-\boldsymbol{W} \prec \mathbf{0} .
$$

2) There exist $\boldsymbol{X} \succ 0, \boldsymbol{W} \succ 0$ and $\boldsymbol{U}$ such that

$$
\left[\begin{array}{cc}
-\boldsymbol{W} & \boldsymbol{V}^{T} \boldsymbol{U}^{T} \\
\boldsymbol{U} \boldsymbol{V} & \boldsymbol{X}-\boldsymbol{U}-\boldsymbol{U}^{T}
\end{array}\right] \prec \mathbf{0} .
$$

\section{FAUlT ESTIMATION STRATEGY}

To make the paper self contained, this section presents the design procedure [11] of the robust observer that will estimate simultaneously the state $\boldsymbol{x}_{k}$, and the fault $\boldsymbol{f}_{k}$, and decouple the effect of the unknown input $\boldsymbol{d}_{k}$. To this purpose, the following structure is proposed

$$
\begin{aligned}
\boldsymbol{z}_{k+1}= & \boldsymbol{N} \boldsymbol{z}_{k}+\boldsymbol{G} \boldsymbol{u}_{k}+\boldsymbol{L} \boldsymbol{y}_{k} \\
& +\boldsymbol{T} \boldsymbol{B} \hat{\boldsymbol{f}}_{k}+\boldsymbol{T} \boldsymbol{g}\left(\hat{\boldsymbol{x}}_{k}, \boldsymbol{u}_{k}\right), \\
\hat{\boldsymbol{x}}_{k}= & \boldsymbol{z}_{k}-\boldsymbol{E} \boldsymbol{y}_{k}, \\
\hat{\boldsymbol{f}}_{k+1}= & \hat{\boldsymbol{f}}_{k}+\boldsymbol{F}\left(\boldsymbol{y}_{k}-\boldsymbol{C} \hat{\boldsymbol{x}}_{k}\right) .
\end{aligned}
$$

The state estimation error can be described by

$$
\begin{aligned}
\boldsymbol{e}_{k} & =\boldsymbol{x}_{k}-\hat{\boldsymbol{x}}_{k}=\boldsymbol{x}_{k}-\boldsymbol{z}_{k}+\boldsymbol{E} \boldsymbol{C} \boldsymbol{x}_{k}+\boldsymbol{E} \boldsymbol{W}_{2} \boldsymbol{w}_{k} \\
& =\boldsymbol{T} \boldsymbol{x}_{k}-\boldsymbol{z}_{k}+\boldsymbol{E} \boldsymbol{W}_{2} \boldsymbol{w}_{k},
\end{aligned}
$$

where $\boldsymbol{T}=\boldsymbol{I}+\boldsymbol{E} \boldsymbol{C}$, and hence

$$
\boldsymbol{z}_{k}=\boldsymbol{T} \boldsymbol{x}_{k}+\boldsymbol{E} \boldsymbol{W}_{2} \boldsymbol{w}_{k}-\boldsymbol{e}_{k}
$$

By (16), the state estimation error is given by

$$
\boldsymbol{e}_{k+1}=\boldsymbol{T} \boldsymbol{x}_{k+1}-\boldsymbol{z}_{k+1}+\boldsymbol{E} \boldsymbol{W}_{2} \boldsymbol{w}_{k+1} \text {. }
$$

By substituting (1) and (13) into (18), the state estimation error dynamics is obtained as follows

$$
\begin{aligned}
\boldsymbol{e}_{k+1} & =\boldsymbol{N} \boldsymbol{e}_{k}+[\boldsymbol{T} \boldsymbol{A}-\boldsymbol{N} \boldsymbol{T}-\boldsymbol{L} \boldsymbol{C}] \boldsymbol{x}_{k} \\
& +\left[\boldsymbol{T} \boldsymbol{W}_{1}-\boldsymbol{N} \boldsymbol{E} \boldsymbol{W}_{2}-\boldsymbol{L} \boldsymbol{W}_{2}\right] \boldsymbol{w}_{k} \\
& +\boldsymbol{T} \boldsymbol{B}\left[\boldsymbol{f}_{k}-\hat{\boldsymbol{f}}_{k}\right]+\boldsymbol{T}\left[\boldsymbol{g}\left(\boldsymbol{x}_{k}, \boldsymbol{u}_{k}\right)-\boldsymbol{g}\left(\hat{\boldsymbol{x}}_{k}, \boldsymbol{u}_{k}\right)\right] \\
& +[\boldsymbol{T} \boldsymbol{B}-\boldsymbol{G}] \boldsymbol{u}_{k}+\boldsymbol{T} \boldsymbol{D} \boldsymbol{d}_{k}+\boldsymbol{E} \boldsymbol{W}_{2} \boldsymbol{w}_{k+1}
\end{aligned}
$$

Subsequently, according to (20), firstly matrix $\boldsymbol{T}$ is determined to satisfy $\boldsymbol{T} \boldsymbol{D}=\mathbf{0}$ and then matrix $\boldsymbol{G}$ is calculated such that $\boldsymbol{G}=\boldsymbol{T} \boldsymbol{B}$ as follows (for further details see [11]).

$$
\begin{aligned}
\boldsymbol{T} \boldsymbol{D} & =\mathbf{0}, \\
\boldsymbol{T} \boldsymbol{A} & =\boldsymbol{N} \boldsymbol{T}+\boldsymbol{L} \boldsymbol{C}, \\
\boldsymbol{T B}-\boldsymbol{G} & =\mathbf{0}, \\
\boldsymbol{s}_{k} & =\boldsymbol{g}\left(\boldsymbol{x}_{k}, \boldsymbol{u}_{k}\right)-\boldsymbol{g}\left(\hat{\boldsymbol{x}}_{k}, \boldsymbol{u}_{k}\right), \\
\boldsymbol{e}_{f, k} & =\boldsymbol{f}_{k}-\hat{\boldsymbol{f}}_{k},
\end{aligned}
$$

leading to

$$
\begin{aligned}
\boldsymbol{e}_{k+1} & =\boldsymbol{N} \boldsymbol{e}_{k}+\boldsymbol{T} \boldsymbol{s}_{k}+\boldsymbol{T} \boldsymbol{B} \boldsymbol{e}_{f, k} \\
& +\left[\boldsymbol{T} \boldsymbol{W}_{1}-\boldsymbol{N} \boldsymbol{E} \boldsymbol{W}_{2}-\boldsymbol{L} \boldsymbol{W}_{2}\right] \boldsymbol{w}_{k}+\boldsymbol{E} \boldsymbol{W}_{2} \boldsymbol{w}_{k+1}
\end{aligned}
$$

Equation (20) can also be expressed in the following form

$$
\begin{aligned}
\boldsymbol{T A} & =\boldsymbol{N}[\boldsymbol{I}+\boldsymbol{E} \boldsymbol{C}]+\boldsymbol{L} \boldsymbol{C}, \\
\boldsymbol{N} & =\boldsymbol{T} \boldsymbol{A}-\boldsymbol{N} \boldsymbol{E} \boldsymbol{C}-\boldsymbol{L} \boldsymbol{C}=\boldsymbol{T A}-\boldsymbol{K} C,
\end{aligned}
$$

where $\boldsymbol{K}=\boldsymbol{N} \boldsymbol{E}+\boldsymbol{L}$. Then, it is obvious that:

$$
\begin{aligned}
\boldsymbol{e}_{k+1} & =\boldsymbol{N} \boldsymbol{e}_{k}+\boldsymbol{T} \boldsymbol{s}_{k}+\boldsymbol{T} \boldsymbol{B} \boldsymbol{e}_{f, k} \\
& +\left[\boldsymbol{T} \boldsymbol{W}_{1}-[\boldsymbol{N} \boldsymbol{E}+\boldsymbol{L}] \boldsymbol{W}_{2}\right] \boldsymbol{w}_{k}+\boldsymbol{E} \boldsymbol{W}_{2} \boldsymbol{w}_{k+1} .
\end{aligned}
$$

Being aware that $\boldsymbol{N} \boldsymbol{E}+\boldsymbol{L}=\boldsymbol{N} \boldsymbol{E}+\boldsymbol{K}-\boldsymbol{N} \boldsymbol{E}=\boldsymbol{K}$, equation (23) can be rewritten as

$$
\begin{aligned}
\boldsymbol{e}_{k+1} & =[\boldsymbol{T} \boldsymbol{A}-\boldsymbol{K} \boldsymbol{C}] \boldsymbol{e}_{k}+\boldsymbol{T} \boldsymbol{s}_{k}+\boldsymbol{T} \boldsymbol{B} \boldsymbol{e}_{f, k} \\
& +\left[\boldsymbol{T} \boldsymbol{W}_{1}-\boldsymbol{K} \boldsymbol{W}_{2}\right] \boldsymbol{w}_{k}+\boldsymbol{E} \boldsymbol{W}_{2} \boldsymbol{w}_{k+1} .
\end{aligned}
$$

Similarly, the fault estimation error is determined by the following relation

$$
\boldsymbol{e}_{f, k+1}=\boldsymbol{f}_{k+1}-\hat{\boldsymbol{f}}_{k+1} .
$$

Using (5) the fault estimation error (25) can be expressed as

$$
\begin{aligned}
\boldsymbol{e}_{f, k+1} & =\boldsymbol{f}_{k+1}-\hat{\boldsymbol{f}}_{k+1} \\
& =\boldsymbol{f}_{k+1}-\hat{\boldsymbol{f}}_{k}-\boldsymbol{F} \boldsymbol{C} \boldsymbol{e}_{k}-\boldsymbol{F} \boldsymbol{W}_{2} \boldsymbol{w}_{k} \\
& =\boldsymbol{f}_{k+1}-\boldsymbol{f}_{k}+\boldsymbol{f}_{k}-\hat{\boldsymbol{f}}_{k}-\boldsymbol{F} \boldsymbol{C} \boldsymbol{e}_{k}-\boldsymbol{F} \boldsymbol{W}_{2} \boldsymbol{w}_{k} \\
& =-\boldsymbol{F} \boldsymbol{C} \boldsymbol{e}_{k}+\boldsymbol{e}_{f, k}+\boldsymbol{\varepsilon}_{f, k}-\boldsymbol{F} \boldsymbol{W}_{2} \boldsymbol{w}_{k} .
\end{aligned}
$$


If the state estimation error dynamics (24) and fault estimation error (26) are given, then the following extended vectors can be introduced

$$
\begin{aligned}
\overline{\boldsymbol{e}}_{k+1} & =\left[\boldsymbol{e}_{k+1}^{T}, \boldsymbol{e}_{f, k+1}^{T}\right]^{T}, \\
\boldsymbol{v}_{k} & =\left[\boldsymbol{w}_{k}^{T}, \boldsymbol{\varepsilon}_{k}^{T}, \boldsymbol{w}_{k+1}^{T}\right]^{T},
\end{aligned}
$$

such that the state and fault estimation error is given by

$$
\begin{aligned}
\overline{\boldsymbol{e}}_{k+1}= & {\left[\begin{array}{cc}
\boldsymbol{T} \boldsymbol{A}-\boldsymbol{K} \boldsymbol{C} & \boldsymbol{T} \boldsymbol{B} \\
-\boldsymbol{F} \boldsymbol{C} & \boldsymbol{I}
\end{array}\right] \overline{\boldsymbol{e}}_{k}+\left[\begin{array}{c}
\boldsymbol{T} \\
\mathbf{0}
\end{array}\right] \boldsymbol{s}_{k} } \\
& +\left[\begin{array}{ccc}
\boldsymbol{T} \boldsymbol{W}_{1}-\boldsymbol{K} \boldsymbol{W}_{2} & \mathbf{0} & \boldsymbol{E} \boldsymbol{W}_{2} \\
-\boldsymbol{F} \boldsymbol{W}_{2} & \boldsymbol{I} & \mathbf{0}
\end{array}\right] \boldsymbol{v}_{k} .
\end{aligned}
$$

This equation can be described by the following compact form

$$
\overline{\boldsymbol{e}}_{k+1}=\boldsymbol{X} \overline{\boldsymbol{e}}_{k}+\boldsymbol{Y} \boldsymbol{s}_{k}+\boldsymbol{Z} \boldsymbol{v}_{k}
$$

where

$$
\begin{aligned}
\boldsymbol{X} & =\overline{\boldsymbol{A}}-\overline{\boldsymbol{K}} \overline{\boldsymbol{C}}=\left[\begin{array}{cc}
\boldsymbol{T} \boldsymbol{A} & \boldsymbol{T} \boldsymbol{B} \\
\mathbf{0} & \boldsymbol{I}
\end{array}\right]-\left[\begin{array}{c}
\boldsymbol{K} \\
\boldsymbol{F}
\end{array}\right]\left[\begin{array}{ll}
\boldsymbol{C} & 0
\end{array}\right], \\
\boldsymbol{Y} & =\left[\begin{array}{c}
\boldsymbol{T} \\
\mathbf{0}
\end{array}\right], \\
Z & =\overline{\boldsymbol{W}}-\overline{\boldsymbol{K}} \overline{\boldsymbol{V}}=\left[\begin{array}{ccc}
\boldsymbol{T} \boldsymbol{W}_{1} & \mathbf{0} & \boldsymbol{E} \boldsymbol{W}_{1} \\
0 & \boldsymbol{I} & \mathbf{0}
\end{array}\right]-\left[\begin{array}{c}
\boldsymbol{K} \\
\boldsymbol{F}
\end{array}\right] \overline{\boldsymbol{W}},
\end{aligned}
$$

with $\overline{\boldsymbol{W}}=\left[\begin{array}{lll}\boldsymbol{W}_{2} & \mathbf{0} & 0\end{array}\right]$. For the purpose of further analysis, let us assume that $\boldsymbol{w}_{k}$ belongs to ellipsoidal sets

$$
\boldsymbol{w}_{k} \in \mathcal{E}_{\boldsymbol{w}}, \mathcal{E}_{\boldsymbol{w}}=\left\{\boldsymbol{w}: \boldsymbol{w}^{T} \boldsymbol{Q}_{\boldsymbol{w}} \boldsymbol{w} \leq 1\right\}, \boldsymbol{Q}_{\boldsymbol{w}} \succ 0 .
$$

From (34) and (5) it is evident that $\boldsymbol{v}_{k} \in \mathcal{E}_{\boldsymbol{v}}$ with

$$
\begin{aligned}
\boldsymbol{v}_{k} \in \mathcal{E}_{\boldsymbol{v}}, \mathcal{E}_{\boldsymbol{v}} & =\left\{\boldsymbol{v}: \boldsymbol{v}^{T} \boldsymbol{Q}_{\boldsymbol{v}} \boldsymbol{v} \leq 1\right\} \\
\boldsymbol{Q}_{\boldsymbol{v}} & =\frac{1}{3} \operatorname{diag}\left(\boldsymbol{Q}_{\boldsymbol{w}}, \boldsymbol{Q}_{\boldsymbol{\varepsilon}}, \boldsymbol{Q}_{\boldsymbol{w}}\right) .
\end{aligned}
$$

For the purpose of further analysis, let consider $\boldsymbol{P} \succ \mathbf{0}$, that allows to use the following definition (cf. [12]):

Definition 1. The system (30) is strictly quadratically bounded with $\boldsymbol{P} \succ \mathbf{0}$ for all allowable $\boldsymbol{v}_{k} \in \mathcal{E}_{\boldsymbol{v}}, k \geq 0$, if $\overline{\boldsymbol{e}}_{k}^{T} \boldsymbol{P} \overline{\boldsymbol{e}}_{k}>1$ implies $\overline{\boldsymbol{e}}_{k+1}^{T} \boldsymbol{P} \overline{\boldsymbol{e}}_{k+1}<\overline{\boldsymbol{e}}_{k}^{T} \boldsymbol{P} \overline{\boldsymbol{e}}_{k}$ for any $\boldsymbol{v}_{k} \in \mathcal{E}_{\boldsymbol{v}}$.

\section{OBSERVER DESIGN}

The main objective of this section is to show the procedure for designing the observer for the system (1)-(2) using the scheme proposed in the previous section. To settle this problem, the above-defined Quadratic Boundedness (QB) [12] is employed.

Theorem 1: The following statements are equivalent:

1) The system (30) is strictly quadratically bounded with $\boldsymbol{P} \succ \boldsymbol{0}$ for all allowable $\boldsymbol{w}_{k} \in \mathcal{E}_{\boldsymbol{Q}}$;
2) There exists $\boldsymbol{N}, \boldsymbol{U}, \boldsymbol{P} \succ \mathbf{0}, 0<\gamma<1, \alpha>0, \beta>0$ such that for all $M \in \mathbb{M}$ the following condition is satisfied:

$$
\left[\begin{array}{ccccc}
\boldsymbol{S}_{1} & * & * & * & * \\
-\alpha \boldsymbol{V} & -\beta \boldsymbol{I} & * & * & * \\
\mathbf{0} & \mathbf{0} & -\gamma \boldsymbol{Q}_{\boldsymbol{v}} & * & * \\
\boldsymbol{P} \overline{\boldsymbol{A}}-\boldsymbol{N} \overline{\boldsymbol{C}} & \boldsymbol{P} \boldsymbol{Y} & \boldsymbol{P} \overline{\boldsymbol{W}}-\boldsymbol{N} \overline{\boldsymbol{V}} & -\boldsymbol{P} & * \\
\boldsymbol{U} \boldsymbol{M} \boldsymbol{V} & \mathbf{0} & \mathbf{0} & \mathbf{0} & \boldsymbol{S}_{2}
\end{array}\right] \prec \mathbf{0}
$$

with $\boldsymbol{S}_{1}=-\boldsymbol{P}+\gamma \boldsymbol{P}+\alpha \boldsymbol{V}^{T}\left(\boldsymbol{M}+\boldsymbol{M}^{T}\right) \boldsymbol{V}^{T}$, $\boldsymbol{S}_{2}=\beta \boldsymbol{I}-\boldsymbol{U}-\boldsymbol{U}^{T}, \boldsymbol{N}=\boldsymbol{P} \overline{\boldsymbol{K}}$.

Proof: Using Definition 1 and the fact that $\boldsymbol{v}_{k}^{T} \boldsymbol{Q}_{\boldsymbol{v}} \boldsymbol{v}_{k} \leq$ 1 (cf. (35)), it is possible to write:

$$
\boldsymbol{v}_{k}^{T} \boldsymbol{Q}_{\boldsymbol{v}} \boldsymbol{v}_{k}<\overline{\boldsymbol{e}}_{k}^{T} \boldsymbol{P} \overline{\boldsymbol{e}}_{k}, \Rightarrow \overline{\boldsymbol{e}}_{k+1}^{T} \boldsymbol{P} \overline{\boldsymbol{e}}_{k+1}-\overline{\boldsymbol{e}}_{k} \boldsymbol{P} \overline{\boldsymbol{e}}_{k}<0 .
$$

As a consequence, using (30) it can be shown that

$$
\begin{aligned}
& \overline{\boldsymbol{e}}_{k+1}^{T} \boldsymbol{P} \overline{\boldsymbol{e}}_{k+1}-\overline{\boldsymbol{e}}_{k}^{T} \boldsymbol{P} \overline{\boldsymbol{e}}_{k}= \\
& \overline{\boldsymbol{e}}_{k}^{T}\left(\boldsymbol{X}^{T} \boldsymbol{P} \boldsymbol{X}-\boldsymbol{P}\right) \overline{\boldsymbol{e}}_{k}+\overline{\boldsymbol{e}}_{k}^{T}\left(\boldsymbol{X}^{T} \boldsymbol{P} \boldsymbol{Y}\right) \boldsymbol{s}_{k}+ \\
& \overline{\boldsymbol{e}}_{k}^{T}\left(\boldsymbol{X}^{T} \boldsymbol{P} \boldsymbol{Z}\right) \boldsymbol{v}_{k}+\boldsymbol{s}_{k}^{T}\left(\boldsymbol{Y}^{T} \boldsymbol{P} \boldsymbol{X}\right) \overline{\boldsymbol{e}}_{k}+ \\
& \boldsymbol{s}_{k}^{T}\left(\boldsymbol{Y}^{T} \boldsymbol{P} \boldsymbol{Y}\right) \boldsymbol{s}_{k}+\boldsymbol{s}_{k}^{T}\left(\boldsymbol{Y}^{T} \boldsymbol{P} \boldsymbol{Z}\right) \boldsymbol{v}_{k}+ \\
& \boldsymbol{v}_{k}^{T}\left(\boldsymbol{Z}^{T} \boldsymbol{P} \boldsymbol{X}\right) \overline{\boldsymbol{e}}_{k}+\boldsymbol{v}_{k}^{T}\left(\boldsymbol{Z}^{T} \boldsymbol{P} \boldsymbol{Y}\right) \boldsymbol{s}_{k}+ \\
& \boldsymbol{v}_{k}^{T}\left(\boldsymbol{Z}^{T} \boldsymbol{P} \boldsymbol{Z}\right) \boldsymbol{v}_{k}<\mathbf{0},
\end{aligned}
$$

and by defining

$$
\overline{\boldsymbol{v}}_{k}=\left[\overline{\boldsymbol{e}}_{k}^{T}, \boldsymbol{s}_{k}^{T}, \boldsymbol{v}_{k}^{T}\right]^{T},
$$

it can be shown that (38) is equivalent to

$$
\overline{\boldsymbol{v}}_{k}^{T}\left[\begin{array}{ccc}
\boldsymbol{X}^{T} \boldsymbol{P} \boldsymbol{X}-\boldsymbol{P} & \boldsymbol{X}^{T} \boldsymbol{P} \boldsymbol{Y} & \boldsymbol{X}^{T} \boldsymbol{P} \boldsymbol{Z} \\
\boldsymbol{Y}^{T} \boldsymbol{P} \boldsymbol{X} & \boldsymbol{Y}^{T} \boldsymbol{P} \boldsymbol{Y} & \boldsymbol{Y}^{T} \boldsymbol{P} \boldsymbol{Z} \\
\boldsymbol{Z}^{T} \boldsymbol{P} \boldsymbol{X} & \boldsymbol{Z}^{T} \boldsymbol{P} \boldsymbol{Y} & \boldsymbol{Z}^{T} \boldsymbol{P} \boldsymbol{Z}
\end{array}\right] \overline{\boldsymbol{v}}_{k} \prec \mathbf{0} .
$$

Following Assumption 1, it is evident that

$$
\boldsymbol{s}_{k}^{T} \boldsymbol{e}_{k} \leq \boldsymbol{e}_{k}^{T} \boldsymbol{M} \boldsymbol{e}_{k}=\frac{1}{2} \boldsymbol{e}_{k}^{T}\left(\boldsymbol{M}+\boldsymbol{M}^{T}\right) \boldsymbol{e}_{k}
$$

By defining

$$
\boldsymbol{V}=\left[\begin{array}{ll}
\boldsymbol{I} & \mathbf{0}
\end{array}\right],
$$

the state estimation error is reflected by

$$
\boldsymbol{e}_{k}=\boldsymbol{V} \overline{\boldsymbol{e}}_{k}=\left[\begin{array}{ll}
\boldsymbol{I} & \mathbf{0}
\end{array}\right]\left[\begin{array}{c}
\boldsymbol{e}_{k} \\
\boldsymbol{e}_{f, k}
\end{array}\right] .
$$

Using (43), inequality (41) can be written as

$$
\boldsymbol{s}_{k}^{T} \boldsymbol{V} \overline{\boldsymbol{e}}_{k} \leq \frac{1}{2} \overline{\boldsymbol{e}}_{k}^{T} \boldsymbol{V}^{T}\left(\boldsymbol{M}+\boldsymbol{M}^{T}\right) \boldsymbol{V} \overline{\boldsymbol{e}}_{k},
$$

which is equivalent to

$$
\frac{1}{2} \overline{\boldsymbol{e}}_{k}^{T} \boldsymbol{V}^{T}\left(\boldsymbol{M}+\boldsymbol{M}^{T}\right) \boldsymbol{V} \overline{\boldsymbol{e}}_{k}-\frac{1}{2} \boldsymbol{s}_{k}^{T} \boldsymbol{V} \overline{\boldsymbol{e}}_{k}-\frac{1}{2} \overline{\boldsymbol{e}}_{k}^{T} \boldsymbol{V}^{T} \boldsymbol{s}_{k} \geq \mathbf{0} .
$$


Thus, for any $\alpha>0$

$$
\alpha \overline{\boldsymbol{v}}_{k}^{T}\left[\begin{array}{ccc}
\boldsymbol{V}^{T}\left(\boldsymbol{M}+\boldsymbol{M}^{T}\right) \boldsymbol{V} & -\boldsymbol{V}^{T} & \mathbf{0} \\
-\boldsymbol{V} & \mathbf{0} & \mathbf{0} \\
\mathbf{0} & \mathbf{0} & \mathbf{0}
\end{array}\right] \overline{\boldsymbol{v}}_{k} \geq \mathbf{0} .
$$

Similarly, from Assumption 2 it can be shown that

$$
\boldsymbol{s}_{k}^{T} \boldsymbol{s}_{k} \leq \boldsymbol{e}_{k}^{T} \boldsymbol{M}^{T} \boldsymbol{M} \boldsymbol{e}_{k},
$$

which is equivalent to

$$
\overline{\boldsymbol{e}}_{k}^{T} \boldsymbol{V}^{T} \boldsymbol{M}^{T} \boldsymbol{M} \boldsymbol{V} \overline{\boldsymbol{e}}_{k}-\boldsymbol{s}_{k}^{T} \boldsymbol{s}_{k} \geq \mathbf{0} .
$$

Thus, for any $\beta>0$

$$
\beta \overline{\boldsymbol{v}}_{k}^{T}\left[\begin{array}{ccc}
\boldsymbol{V}^{T} \boldsymbol{M}^{T} \boldsymbol{M} \boldsymbol{V} & \mathbf{0} & \mathbf{0} \\
\mathbf{0} & -\boldsymbol{I} & \mathbf{0} \\
\mathbf{0} & \mathbf{0} & \mathbf{0}
\end{array}\right] \overline{\boldsymbol{v}}_{k} \geq \mathbf{0}
$$

From (37), in particular from $\boldsymbol{v}_{k}^{T} \boldsymbol{Q}_{\boldsymbol{v}} \boldsymbol{v}_{k}<\overline{\boldsymbol{e}}_{k}^{T} \boldsymbol{P} \overline{\boldsymbol{e}}_{k}$ it is evident that for $\gamma>0$

$$
\gamma \overline{\boldsymbol{v}}_{k}^{T}\left[\begin{array}{ccc}
-\boldsymbol{P} & \mathbf{0} & \mathbf{0} \\
\mathbf{0} & \mathbf{0} & \mathbf{0} \\
\mathbf{0} & \mathbf{0} & \boldsymbol{Q}_{\boldsymbol{v}}
\end{array}\right] \overline{\boldsymbol{v}}_{k} \prec \mathbf{0} .
$$

Now, using the S-procedure for (40), (45), (48) and (49) it can be concluded that

$$
\overline{\boldsymbol{v}}_{k}^{T}\left[\begin{array}{ccc}
\boldsymbol{S}_{3} & * & * \\
\boldsymbol{Y}^{T} \boldsymbol{P} \boldsymbol{X}-\alpha \boldsymbol{V} & \boldsymbol{S}_{4} & * \\
\boldsymbol{Z}^{T} \boldsymbol{P} \boldsymbol{X} & \boldsymbol{Z}^{T} \boldsymbol{P} \boldsymbol{Y} & \boldsymbol{S}_{5}
\end{array}\right] \overline{\boldsymbol{v}}_{k} \prec \mathbf{0}
$$

where $\boldsymbol{S}_{3}=\boldsymbol{X}^{T} \boldsymbol{P} \boldsymbol{X}-\boldsymbol{P}+\gamma \boldsymbol{P}+\alpha \boldsymbol{V}^{T}\left(\boldsymbol{M}+\boldsymbol{M}^{T}\right) \boldsymbol{V}+$ $\beta \boldsymbol{V}^{T} \boldsymbol{M}^{T} \boldsymbol{M} \boldsymbol{V}, \boldsymbol{S}_{4}=\boldsymbol{Y}^{T} \boldsymbol{P} \boldsymbol{Y}-\beta \boldsymbol{I}, \boldsymbol{S}_{5}=\boldsymbol{Z}^{T} \boldsymbol{P} \boldsymbol{Z}-\gamma \boldsymbol{Q}_{\boldsymbol{v}}$. Rewriting (50) as

$$
\left[\begin{array}{l}
\boldsymbol{X}^{T} \\
\boldsymbol{Y}^{T} \\
\boldsymbol{Z}^{T}
\end{array}\right] \boldsymbol{P}\left[\begin{array}{lll}
\boldsymbol{X} & \boldsymbol{Y} & \boldsymbol{Z}
\end{array}\right]+\left[\begin{array}{ccc}
\boldsymbol{S}_{6} & * & * \\
-\alpha \boldsymbol{V} & -\beta \boldsymbol{I} & * \\
\mathbf{0} & \mathbf{0} & -\gamma \boldsymbol{Q}_{\boldsymbol{v}}
\end{array}\right] \prec \mathbf{0}
$$

with $\boldsymbol{S}_{6}=\boldsymbol{S}_{1}+\beta \boldsymbol{V}^{T} \boldsymbol{M}^{T} \boldsymbol{M} \boldsymbol{V}$ and then applying Schur complements to (51) and next Lemma 1 and then substituting

$$
\begin{aligned}
P X & =P \bar{A}-P \bar{K} \bar{C}=P \bar{A}-\boldsymbol{N} \bar{C}, \\
P Z & =P \bar{W}-P \bar{K} \bar{V}=P \bar{W}-\boldsymbol{N} \bar{V},
\end{aligned}
$$

where $\boldsymbol{N}=\boldsymbol{P} \overline{\boldsymbol{K}}$, lead to (36), which completes the proof.

\section{CONFIDENCE INTERVAlS DESigN}

A design procedure to overbound the unknown real state and fault is proposed in this section.

\section{A. Confidence intervals}

To solve the above problem, let us start with the following theorem:

Theorem 2: If the system (30) is strictly quadratically bounded for all $\boldsymbol{v}_{k} \in \mathcal{E}_{\boldsymbol{v}}$, then the confidence intervals for the state and fault are given as follows:

$$
\begin{gathered}
\hat{\boldsymbol{x}}_{i, k}-z_{i, k} \leq \boldsymbol{x}_{i, k} \leq \hat{\boldsymbol{x}}_{i, k}+z_{i, k}, \quad i=1, \ldots, n, \\
\hat{\boldsymbol{f}}_{j, k}-z_{i, k} \leq f_{j, k} \leq \hat{\boldsymbol{f}}_{j, k}+z_{i, k}, \quad j=1, \ldots, r, \\
i=n+1, \ldots, n+r,
\end{gathered}
$$

with

$$
\begin{aligned}
z_{i, k} & =\left(\zeta_{k}(\gamma) \boldsymbol{c}_{i}^{T} \boldsymbol{P}^{-1} \boldsymbol{c}_{i}\right)^{\frac{1}{2}} \\
\zeta_{k}(\gamma) & =(1-\gamma)^{k} V_{0}+1-(1-\gamma)^{k}, \quad k=0,1, \ldots
\end{aligned}
$$

where $V_{k}=\overline{\boldsymbol{e}}_{k}^{T} \boldsymbol{P} \overline{\boldsymbol{e}}_{k}$ and $\boldsymbol{c}_{i}$ is the $i$ th column of an $n+r$ order identity matrix.

Proof: Theorem 1 guarantees that there exist $\gamma \in(0,1)$ and $\boldsymbol{P} \succ \mathbf{0}$ such that (34) holds. Moreover, from (50) and (40) it can be shown that for all $M \in \mathbb{M}$

$$
\begin{aligned}
V_{k+1} & <(1-\gamma) V_{k}- \\
\alpha & \left(\overline{\boldsymbol{e}}_{k}^{T} \boldsymbol{V}^{T}\left(\boldsymbol{M}+\boldsymbol{M}^{T}\right) \boldsymbol{V} \overline{\boldsymbol{e}}_{k}-\boldsymbol{s}_{k}^{T} \boldsymbol{V} \overline{\boldsymbol{e}}_{k}-\overline{\boldsymbol{e}}_{k}^{T} \boldsymbol{V}^{T} \boldsymbol{s}_{k}\right) \\
& -\beta\left(\overline{\boldsymbol{e}}_{k}^{T} \boldsymbol{V}^{T} \boldsymbol{M}^{T} \boldsymbol{M} \boldsymbol{V} \overline{\boldsymbol{e}}_{k}-\boldsymbol{s}_{k}^{T} \boldsymbol{s}_{k}\right)+\gamma \boldsymbol{v}_{k}^{T} \boldsymbol{Q}_{v} \boldsymbol{v}_{k},
\end{aligned}
$$

Subsequently, by (44) and (47) as well as by the fact that $\boldsymbol{v}_{k}^{T} \boldsymbol{Q}_{v} \boldsymbol{v}_{k} \leq 1$, the upper bound of $V_{k+1}$ defined by (55) can be overbounded with the non-strict inequality of the form

$$
V_{k+1} \leq \gamma+(1-\gamma) V_{k}
$$

Following [12], by induction, inequality (56) yields

$$
V_{k} \leq \zeta_{k}(\gamma), \quad k=0,1, \ldots,
$$

where the sequence $\zeta_{k}(\gamma)$ is defined by (54). Thus, from (57) it is evident that for any $\boldsymbol{v}_{k} \in \mathcal{E}_{\boldsymbol{v}}, \overline{\boldsymbol{e}}_{k}$ is contained inside the ellipsoid

$$
\overline{\boldsymbol{e}}_{k}^{T} \boldsymbol{P} \overline{\boldsymbol{e}}_{k} \leq \zeta_{k}(\gamma)
$$

The maximum and minimum values of $\overline{\boldsymbol{e}}_{i, k}$ can be computed by maximizing/minimizing $\boldsymbol{c}_{i}^{T} \overline{\boldsymbol{e}}_{k}$ under (58). Using the Lagrange approach, the following Lagrange function can be formulated

$$
\boldsymbol{h}\left(\overline{\boldsymbol{e}}_{k}, \lambda\right)=\boldsymbol{c}_{i}^{T} \overline{\boldsymbol{e}}_{k}+\lambda\left(\overline{\boldsymbol{e}}_{k}^{T} \boldsymbol{P} \overline{\boldsymbol{e}}_{k}-\zeta_{k}(\gamma)\right),
$$

where $\lambda$ stands for the Lagrange multiplier. Differentiating (59) with respect to $\bar{e}_{k}$ and $\lambda$ yields

$$
\begin{aligned}
& \frac{\partial \boldsymbol{h}\left(\overline{\boldsymbol{e}}_{k}, \lambda\right)}{\partial \overline{\boldsymbol{e}}_{k}}=\boldsymbol{c}_{i}^{T}+2 \lambda \overline{\boldsymbol{e}}_{k}^{T} \boldsymbol{P}=\mathbf{0}, \\
& \frac{\partial \boldsymbol{h}\left(\overline{\boldsymbol{e}}_{k}, \lambda\right)}{\partial \lambda}=\overline{\boldsymbol{e}}_{k}^{T} \boldsymbol{P} \overline{\boldsymbol{e}}_{k}-\zeta_{k}(\gamma)=0 .
\end{aligned}
$$

Thus, from (60), it can be shown that

$$
\overline{\boldsymbol{e}}_{k}^{T}=-\frac{1}{2 \lambda} \boldsymbol{c}_{i}^{T} \boldsymbol{P}^{-1} \text {. }
$$


Substituting (62) into (61) leads to

$$
\lambda= \pm \frac{1}{2}\left(\zeta_{k}(\gamma)^{-1} \boldsymbol{c}_{i}^{T} \boldsymbol{P}^{-1} \boldsymbol{c}_{i}\right)^{\frac{1}{2}}
$$

Finally, introducing (63) into (62) yields

$$
-z_{i, k} \leq \overline{\boldsymbol{e}}_{i, k} \leq z_{i, k}, \quad i=1, \ldots, n+r,
$$

where $z_{i, k}$ is given by (53), which completes the proof.

Note that (52) can be perceived as confidence intervals for $\boldsymbol{x}_{k}$ and $\boldsymbol{f}_{k}$, respectively.

\section{B. Final design procedure}

As it was already mentioned in the previous section, $\zeta_{k}$ converges exponentially to one, while the convergence depends on $\gamma$. Thus, the steady state length of (52) depends solely on the eigenvalues of $\boldsymbol{P}$, which describe the size of the ellipsoid. In this paper, the A-optimality [18] criterion is selected such that the following optimization problem is obtained

$$
\max _{\boldsymbol{N}, \boldsymbol{U}, \boldsymbol{P} \succ \mathbf{0}, 0<\gamma<1, \alpha>0, \beta>0} \operatorname{Trace}(\boldsymbol{P}),
$$

for all $M \in \mathbb{M}$ under the constraints formed with (36).

\section{CASE STUdy}

The proposed approach is applied to a twin rotor MIMO [19]. The nonlinear model has been discretized using Euler method with sampling time $T_{s}=0.01 \mathrm{~s}$, such that it can be expressed as (1)-(2) around an equilibrium point $\boldsymbol{x}_{e q}$, as follows

$$
\begin{aligned}
\boldsymbol{x}_{k+1} & =\boldsymbol{A}\left(\boldsymbol{x}_{e q}\right) \boldsymbol{x}_{k}+\boldsymbol{B}\left(\boldsymbol{x}_{e q}\right) \boldsymbol{u}_{k}+\boldsymbol{D} \boldsymbol{d}_{k}+\boldsymbol{g}\left(\boldsymbol{x}_{e q}, \boldsymbol{x}_{k}, \boldsymbol{u}_{k}\right) \\
& +\boldsymbol{B}\left(\boldsymbol{x}_{e q}\right) \boldsymbol{f}_{k}+\boldsymbol{W}_{1} \boldsymbol{w}_{k}, \\
\boldsymbol{y}_{k} & =\boldsymbol{C} \boldsymbol{x}_{k}+\boldsymbol{W}_{2} \boldsymbol{w}_{k}
\end{aligned}
$$

where $\boldsymbol{A}\left(\boldsymbol{x}_{e q}\right)$ and $\boldsymbol{B}\left(\boldsymbol{x}_{e q}\right)$ are the frozen system matrices at the equilibrium point, while the non-linear function $\boldsymbol{g}\left(\boldsymbol{x}_{e q}, \boldsymbol{x}_{k}, \boldsymbol{u}_{k}\right)$ is defined as

$$
\boldsymbol{g}\left(\boldsymbol{x}_{e q}, \boldsymbol{x}_{k}, \boldsymbol{u}_{k}\right)=\left(\boldsymbol{A}\left(\boldsymbol{x}_{k}\right)-\boldsymbol{A}\left(\boldsymbol{x}_{e q}\right)\right) \boldsymbol{x}_{k} .
$$

In order to illustrate the proposed estimation scheme, it will be assumed that some state variables are not directly measured. The detailed description of the model is given in [11] and is omitted due the lack of space.

Let the initial condition for the system and the observer be $\boldsymbol{x}_{0}=[0.1,0.2,0.5,0.1,0.2,0.5]^{T}, \boldsymbol{z}_{0}=\mathbf{0}, \hat{\boldsymbol{f}}_{0}=\mathbf{0}$, while the input

$$
u_{h}(k)=0.5 \cos \left(\frac{k}{50}\right), \quad u_{v}(k)=0.2 \sin \left(\frac{k}{80}\right),
$$

and constant input disturbance $d_{k}=1$ have been considered, and $\boldsymbol{w}_{k}$ has been chosen as a uniformly distributed random vector, where each element takes values in the interval $[-0.1,0.1]$. Moreover, let us consider the following fault scenarios i.e., additive faults in the tail motor $f_{h}(k)$ and additive faults in the main motor $f_{v}(k)$, described as follows:
Fault scenario $\mathrm{S} 1$ :

$$
\begin{aligned}
& f_{h}(k)=\left\{\begin{array}{cc}
-0.4 & 50 \leq k \leq 400, \\
0 & \text { otherwise }
\end{array}\right. \\
& f_{v}(k)=\left\{\begin{array}{cc}
-0.6 & 50 \leq k \leq 400, \\
0 & \text { otherwise }
\end{array}\right.
\end{aligned}
$$

Fault scenario S2:

$$
\begin{aligned}
& f_{h}(k)=\left\{\begin{array}{cc}
-0.003(k-400) & 50 \leq k \leq 400 \\
0 & \text { otherwise }
\end{array}\right. \\
& f_{v}(k)=\left\{\begin{array}{cc}
0.002(k-400) & 50 \leq k \leq 400 \\
0 & \text { otherwise }
\end{array}\right.
\end{aligned}
$$

The analysis starts with the fault-free case, i.e., $f_{h}(k)=0$ and $f_{h}(k)=0$. Figure 1 shows the resulting $\operatorname{Trace}(\boldsymbol{P})$ for $\gamma \in(0,1)$ and $\boldsymbol{Q}_{\boldsymbol{v}}=\operatorname{diag}\left[0.05 \boldsymbol{I}^{n \times n}, 10 \boldsymbol{I}^{r \times r}, 0.05 \boldsymbol{I}^{n \times n}\right]$ after solving (38). As it can be observed, the size of the ellipsoid increases when the variable $\gamma$ increases, which clearly shows that the larger is $\gamma$, the faster is the response of the estimator. Figure 1 shows, that maximum trace of matrix $\boldsymbol{P}$ is for $\gamma=0.7722$. This is caused by infeasibility of the LMIs for $\gamma>0.7722$. For the further study $\gamma=$ 0.7 was selected. Figs. $2-4$ present the state (black line) and its estimate (red line) with adaptive threshold (green line) for S1. From these results, it is evident that the state estimation is performed with a good quality even when some state variables are not directly measured. It It can also be mentioned that the adaptive threshold can be perceived as a $100 \%$ confidence interval of the state estimate. Finally, Figs. 5-6 show fault estimates for S1 and S2. It can be seen that the fault is estimated with a satisfactory accuracy.

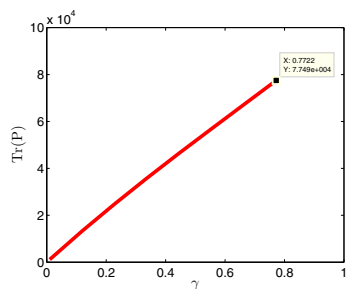

Fig. 1. Evolution of trace value of matrix $\boldsymbol{P}$ for $\gamma \in(0,1)$
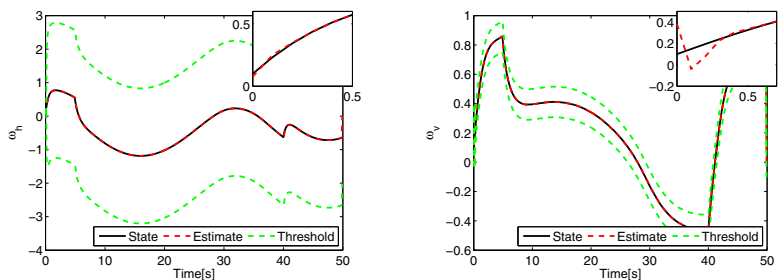

Fig. 2. State variables $\omega_{h}$ (velocity of the tail rotor) and $\omega_{v}$ (velocity of the main rotor) (black line) and their estimates (red line) and adaptive threshold (green line) for $k=0, \ldots, 50$ for $\mathrm{S} 1$.

\section{CONCLUSIONS}

A robust adaptive fault estimation with unknown input decoupling for nonlinear system has been proposed. The 

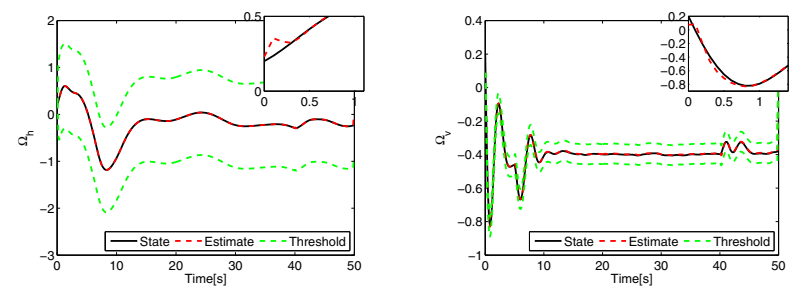

Fig. 3. State variables $\Omega_{h}$ (the angular velocity of the TRMS around the vertical axis) and $\Omega_{v}$ (the angular velocity of the TRMS around the horizontal axis) (black line) and their estimates (red line) and adaptive threshold (green line) for $k=0, \ldots, 50$ for $\mathrm{S} 1$.
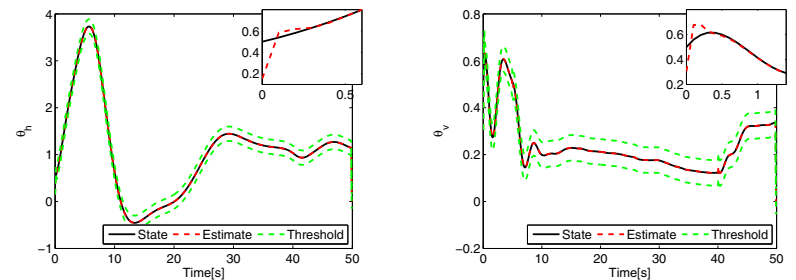

Fig. 4. State variables $\theta_{h}$ (the yaw angle of the beam) and $\theta_{v}$ (the pitch angle of the beam) (black line) and their estimates (red line) and adaptive threshold (green line) for $k=0, \ldots, 50$ for $\mathrm{S} 1$.
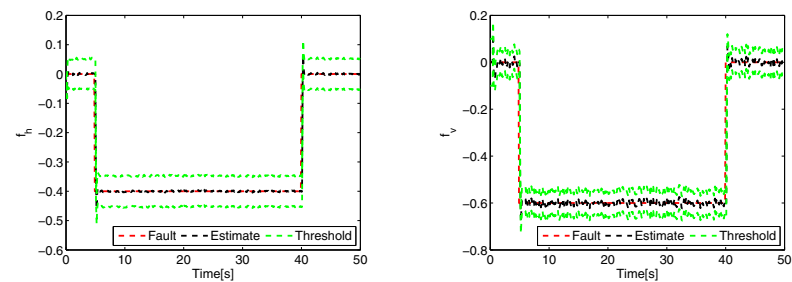

Fig. 5. Fault $f_{h}$ (the tail motor) and $f_{v}$ (the main motor) (red line) and their estimates (black line) and adaptive threshold (green line) for $k=0, \ldots, 50$ for $\mathrm{S} 1$.
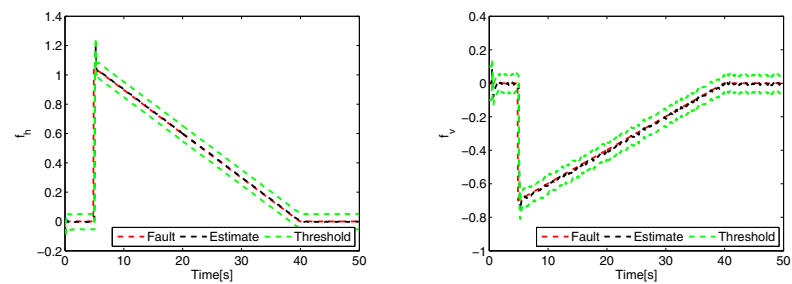

Fig. 6. Fault $f_{h}$ (the tail motor) and $f_{v}$ (the main motor) (red line) and their estimates (black line) and adaptive threshold (green line) for $k=0, \ldots, 50$ for $\mathrm{S} 2$.

quadratic boundedness approach has been used to design the robust observer to simultaneously estimate the state and fault as well as decoupling the effect of an unknown input. The proposed design procedure is relatively simple and boils down to solving a set of linear matrix inequalities. The confidence intervals overbounding the true state and fault has been proposed as well. Finally, the paper has shown an illustrative example demonstrating the effectiveness of the proposed approach in the fault estimation for nonlinear system.

\section{ACKNOWLEDGMENT}

The work was supported by the National Science Centre of Poland under grant: 2013/11/B/ST7/01110. The authors also acknowledge support by the Spanish Ministry of Science and Technology through the projects CICYT ECOCIS (ref. DPI2013-48243-C2-1-R) and CICYT HARCRICS (ref. DPI2014-58104-R), by AGAUR through the contract FIDGR 2015 (ref. 2015FI_B2_00171), and by the DGR of the Generalitat de Catalunya (ref. 2014/SGR/374)

\section{REFERENCES}

[1] J. Korbicz, J. Kościelny, Z. Kowalczuk, and W. Cholewa (Eds.), Fault Diagnosis. Models, Artificial Intelligence, Applications. Berlin: Springer-Verlag, 2004.

[2] M. Witczak, Modelling and Estimation Strategies for Fault Diagnosis of Non-linear Systems. Berlin: Springer-Verlag, 2007.

[3] J. Y. Keller and M. Darouach, "Two-stage Kalman estimator with unknown exogenous inputs." Automatica, vol. 35, no. 2, pp. 339-342, 1999.

[4] S. Gillijns and B. De Moor, "Unbiased minimum-variance input and state estimation for linear discrete-time systems," Automatica, vol. 43, pp. 111-116, 2007.

[5] X. Zhang, M. Polycarpou, and T. Prisini, "Fault diagnosis of a class of nonlinear uncertain systems with Lipschitz nonlinearities using adaptive estimation," Automatica, vol. 46, no. 2, pp. 290-299, 2010.

[6] K. Veluvolu, M. Kim, and D. Lee, "Nonlinear sliding mode highgain observers for fault estimation," International Journal of Systems Science, vol. 42, no. 7, pp. 1065-1074, 2011.

[7] E. Nobrega, M. Abdalla, and K. Grigoriadis, "Robust fault estimation of unceratain systems using an LMI-based approach," International Journal of Robust and Nonlinear Control, vol. 18, no. 7, pp. 16571680, 2008.

[8] L. Seybold, M. Witczak, P. Majdzik, and R. Stetter, "Towards robust predictive fault-tolerant control for a battery assembly system," International Journal of Applied Mathematics and Computer Science, vol. 25, no. 4, pp. 849-862, 2015.

[9] H. Hammouri, P. Kabore, S. Othman, and J. Biston, "Failure diagnosis and nonlinear observer. application to a hydraulic process," Journal of The Franklin Institute, vol. 339, no. 4-5, pp. 455-478, 2002.

[10] C. D. Persis and A. Isidori, "A geometric approach to nonlinear fault detection and isolation," Automatic Control, IEEE Transactions on, vol. 46, no. 6, pp. 853-865, 2001.

[11] M. Witczak, M. Buciakowski, V. Puig, D. Rotondo, and F. Nejjari, "An lmi approach to robust fault estimation for a class of nonlinear systems," International Journal of Robust and Nonlinear Control, vol. 26, no. 7, pp. 1530-1548, 2006.

[12] A. Alessandri, M. Baglietto, and G. Battistelli, "Design of state estimators for uncertain linear systems using quadratic boundedness," Automatica, vol. 42, no. 3, pp. 497-502, 2006.

[13] A. Zemouche, M. Boutayeb, and G. Iulia Bara, "Observer for a class of Lipschitz systems with extension to $\mathcal{H}_{\infty}$ performance analysis,' Systems and Control Letters, vol. 57, no. 1, pp. 18-27, 2008.

[14] A. Zemouche and M. Boutayeb, "Observer design for Lipschitz nonlinear systems: the discrete time case," IEEE Transactions on Circuits and Systems - II:Express Briefs, vol. 53, no. 8, pp. 777-781, 2006.

[15] M. Abbaszadeh and H. Marquez, "LMI optimization approach to robust $\mathcal{H}_{\infty}$ observer design and static output feedback stabilization for non-linear uncertain systems," International Journal of Robust and Nonlinear Control, vol. 19, no. 3, pp. 313-340, 2008.

[16] H. Marquez, Nonlinear control systems. Analysis and Design. New Jersey: Wiley, 2003.

[17] M. de Oliveira, J. Bernussou, and J. Geromel, "A new discrete-time robust stability condition," Systems and Control Letters, vol. 37, no. 4, pp. 261-265, 1999.

[18] M. Witczak, Fault Diagnosis and Fault-Tolerant Control Strategies for Non-Linear Systems: Analytical and Soft Computing approaches. Heidelberg, Germany: Springer International Publishing, 2014.

[19] D. Rotondo, F. Nejjari, and V. Puig, "Quasi-LPV modeling, identification and control of a twin rotor MIMO system," Control Engineering Practice, vol. 21, no. 6, p. 829846, 2013. 